\title{
A driver's car-following behavior prediction model based on multi-sensors data
}

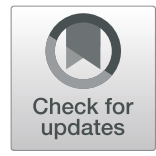

\author{
Hui Wang, Menglu Gu, Shengbo Wu and Chang Wang* (D)
}

\begin{abstract}
The prerequisite for the effective operation of vehicle collision warning system is that the necessary operation is not implemented. Therefore, the behavior prediction that the driver should perform when the preceding vehicle braking is the key to improve the effectiveness of the warning system. This study was conducted to acquire characteristics in the car-following behavior when confronted by the braking of the preceding vehicle, including the reaction time and operation behavior, and establish a behavior prediction model. A driving experiment on the expressway was conducted using devices, such as millimeter-wave radars and controller area network (CAN) bus data, to acquire 845 segments of car following when the brake lamps of the car ahead are on. Data analysis demonstrates that the mean of time distance of car following, mean of car-following distance, and time-to-collision (TTC) mean are closely related with whether or not the driver slowed the car down. The operation states of the driver were divided into keeping the unchanged state of the degree of accelerator pedal opening, loosening of accelerator pedal without braking, braking, and other special situations with the input variables of car-following distance, speed of driver's car, relative speed, time distance, and TTC using the support vector machine (SVM) method to build a prediction model for the operation behavior of the driver. The verification result showed that the model predicts driving behavior with an accuracy rate of $80 \%$. It reflects the actual decision-making process of the driver, especially the normal operation of the driver, to loosen the accelerator pedal without braking. This model can help to optimize the algorithm of the rear-end accident warning system and improve intelligent system acceptance.
\end{abstract}

Keywords: Machine learning, Car following, Sensor data, Prediction model, Time-to-collisionAbbreviations, CAN Controller area network, GMM Gaussian mixed model, GPS Global position system, IPC Industrial personal computer, ROC Receiver operating characteristic curve, SVM Support vector machine, THW Time headway, TTC Time-tocollision

\section{Introduction}

Rear-end accidents are the most common type of traffic accident. Statistical data for related accidents illustrate that the rear-end accident is the most frequent accident type among all accidents on the expressway, having a serious impact on traffic and usually ending in severe consequences [1]. An analysis of the process of rear-end accidents is divided into two situations. The first situation is when carfollowing distance is so close that when the car ahead brakes, a rear-end accident may occur at a long braking distance even if the driver brakes in time. In the second situation, the car-following distance of the driver is relatively

\footnotetext{
* Correspondence: wangchang@chd.edu.cn

School of Automobile, Chang'an University, Middle Section of Nan'er Huan Road, Xi'an 710064, Shaanxi, China
}

safe, but when the car ahead brakes, the driver is in a state of distraction or fatigue and that individual does not notice the braking of the car ahead. This situation is similar to the one where braking does not occur at all.

The occurrence rates of these two kinds of rear-end accidents are comparatively higher. Thus, some vehicles have begun promoting the use of a rear-end collision alarm system. Rear-end collision alarm systems can monitor the moving state of the car ahead using radars or visual sensors and provide a warning when there is danger. Due to different driving styles of drivers during car following, too frequent alarms will cause a feeling of antipathy of some drivers [2]. Thus, these systems usually set the alarm time when the car is already in a comparatively dangerous state to reduce the distraction of the driver. However, the time of 
warning has a great influence on the effectiveness of the warning system. A later warning time may increase the rate of accidents [3].

Numerous studies have been conducted from different perspectives in the security of car following. Kometani [4] proposed a car-following model based on the safety distance; it means the minimum distance to avoid collision when the preceding vehicle starts emergency braking. Safety distance is the basis of the general motor model which consists of relative speed and distance [5]. Based on the general motor model, Treitere et al. [6] divided the car-following into two phases: acceleration and deceleration. Furthermore, Aron [7] divided the car-following into three stages: acceleration, deceleration, and keeping. Helly [8] suggested a linear model that consists of the acceleration, relative speed, and distance. Based on the research on the desired spacing distance, Pete [9] proposed a desired spacing model. Some studies focused on the car-following model with respect to the speed and car-following distance. Jiang et al. conducted a motorcade following the experiment and investigated the relationship between car speed and car-following distance in different traffic environments [10]. Tang et al. suggested that forward and backward safe distance should be taken into consideration while following a car; the result showed that the models based on the two kinds of safe distances were better than the other models [11]. Gipps proposed a car-following model of the driver through a simulation and performed a correlation analysis with actual traffic flow parameters, offering a theoretical foundation for follow-up studies on car following [12]. Zhou et al. provided a more stable carfollowing model by considering the moving features of the car ahead [13]. Tang et al. found that the headway is an important parameter that affects the risk assessment of the driver [14-16]. Furthermore, based on the time headway (THW) and driving speed, Simons-Morton et al. provided a method that can identify the age and gender of the driver [17]. Trnros et al. and Varotto et al. investigated investigated the impact of an adaptive cruise control (ACC) system on the TTC and driving speed $[18,19]$.

However, these studies have provided many car-following models from the perspective of safe car-following distance and the state of the leading vehicle movement. The influence of the reaction from the driver on car following was underestimated. Chung established a deceleration model with respect to the influence of acceleration on the following vehicle and suggested that the response time of the driver to the brake of the leading car was an important factor affecting potential accidents. Many studies have pointed out that the reaction time of the drivers varies greatly and drivers of different ages have significantly different reaction times when driving [20]. Further, the reaction time of the driver increases under fatigue driving [21, 22]. In distracted driving, the reaction time of the driver and the following distance obviously fluctuated [23-25]. Owing to the uncertainty of the relative speed and reaction time of the driver during car following, Sheu provided a car-following model based on quantum mechanics, and the model was verified by conducting an experiment on a driving simulator [26]. Li et al. confirmed the uncertainty of the response time of the driver while following a car and built a stable car-following model that could effectively alleviate traffic congestion [27]. Yang observed real traffic flow in a set road section and acquired the car-following data of drivers. The analysis of the delayed time and anticipated distance revised the car-following model and improved its effectiveness [28].

Although the reaction time of the driver was considered in these models, the reaction time of the same driver is not exactly the same in different situations $[29,30]$. Thus, some scholars suggested that a driving model should consider the operation of the driver. Wakita et al. focused on the influence of the accelerator pedal during car following, and a driver identification method was established by using Gaussian mixed model (GMM) in which the identification rate reached 73\% [31]. Based on the vehicle speed and acceleration, Kumagai established a driving model to predict whether the driver could stop in front of the red lamps with Dynamic Bayesian Networks [32]. In addition, fuzzy neural networks and fuzzy inference methods have been widely used in driver behavior analyses [33, 34]. Among them, the support vector machine (SVM) has a good application effect. SVM has been used in many fields, such as pattern recognition, regression, and equalization [35-37]. Based on SVM, Qian et al. provided a driver identification method with data of steering, brake, and acceleration pedals [38]. Some studies carried on the field test and proposed a driving model to identify the driving intention of the driver by using the SVM $[39,40]$.

Many models of car-following behavior discussed and analyzed the influential factors of models in car-following behavior. Most present models focused on the characteristic parameter of normal car-following process. The most dangerous moment in rear-end accident process begins when the stop lamps of the car ahead are on. The behavior of the driver at this moment directly influences the seriousness of the accident and whether rear-end accident will occur. Therefore, if the operation of the driver and moving state of the car ahead can be monitored in real time, while the braking of the car ahead and driver do not take any operation, warning time can be adjusted in advance to guarantee effectiveness. An early warning algorithm should take the actual operations of the driver while driving into consideration. Early warning will only be triggered when the driver needs to take operation but they fail to do so. It is necessary to adjust the timing of the early warning with respect to the driving behavior during car following, maintaining car-following safety [41].

Most researches of car-following emphasized on the relationship of host car speed, relative speed, and distance; 
however, the impact of driver's reaction on brake lamps on car following is still unclear. Drivers consider they are in a safe driving situation when the preceding vehicle is normal driving, but their risk awareness will increase rapidly once the brake lamps of the car ahead turn on. At this time, the driving behavior is different from that in the traditional follow-up model.

Therefore, this study mainly focuses on investigating the response of the driver when the car ahead starts to brake, including the reaction time and operation behavior during the car-following process. To acquire the carfollowing characteristics of the driver, we conducted a road test with an equipment vehicle. Several segments of brake of the car ahead were selected. We collected the kinematics parameters of the host car and the preceding vehicle during car-following processes, and four different operation behaviors were classified according to the data analysis. Finally, the behavior prediction model of the driver when the brake lamps of the car ahead were on was established based on the SVM method. This behavior prediction model can predict the actions that the driver should take when confronted by the braking behavior of the preceding vehicle based on the current state of the host vehicle and the preceding vehicle's kinetic information. By comparing predictive behavior with actual behavior and when the difference was obviously appeared, which indicated that the driver's operation at this time possessed certain risks, it was necessary to give an early warning of his current driving behavior. In this way, the collision warning system could transmit a warning based on the specific driving behavior and reduce the interference of frequent warnings to drivers. Hence, the proposed model can help to optimize the algorithm of the rear-end accident warning system and improve the intelligent system acceptance.

\section{Methods}

This study organized several participants for a real road driving test by building a real car driving platform with related sensors. This approach was adopted to acquire the car-following data of the driver and determine state data when the brake lamps of the car ahead were on. After the test, related data was combined with video monitoring system data when the brake lamps of the car ahead were on screen. This approach provided precise data support for follow-up analysis and modeling. Based on the analysis of parameter characteristics, a prediction model of driver's car-following behavior was established by using the SVM method.

\subsection{Test instruments}

The test vehicle is a Volkswagen multi-purpose vehicle. Millimeter-wave radar, global positioning system, multichannel video monitoring system, and bus acquisition card for controller area network were installed on the multi-purpose vehicle platform to acquire and record driving data. The installation of these devices did not affect the normal operation of the driver. Millimeterwave radar was used to collect the relative distance and velocity between the host vehicle and the preceding vehicle. Video monitoring system was employed to record the braking behavior of the preceding vehicle. GPS device provided the geographical positions and velocity of the host vehicle. CAN bus served as the data transmission channel, and IPC stored all the collected data (Table 1). Figure 1 shows the composition of the testing system.

\subsection{Test road}

Rear-end accidents are typical on expressways. Thus, this study utilizes the expressway as test road. A closed two-way, six-lane freeway was selected for the driving experiments. The traffic flow on the route chosen should be heavy enough for subjecting the driver to the carfollowing state for most of the time to acquire sufficient car-following data. Therefore, an expressway was chosen for the real car test with a speed limit of $110 \mathrm{~km} / \mathrm{h}$ and a distance of $40.5 \mathrm{~km}$. Traffic flow was heavy during the test. Two lanes were used in one direction. The driver was in the state of car following for most of the time to generate a significant amount of car-following data.

\subsection{Participant driver}

Twenty participant drivers were recruited (17 males, 3 females), and none of them were professional drivers. The age of participants ranged from 27 to 50 years with a mean age of 40.6 years. The driving years of the participants ranged from 2 to 30 years with a mean driving year of 14.6. The participants were physically healthy, and none had been involved in a severe traffic accident in the past 5 years. All participants could drive the trial car independently and safely with visual acuity (including corrected visual acuity) above 1.0 in both eyes. They were in good physical condition and full of energy during the test.

\subsection{Test process}

Before the test, the participants were informed they needed to drive the test vehicle as usual during the entire test, and they should drive the car from the starting point to the specified toll station, then drive to the exit from the toll station of the expressway and back to the starting point. During the driving test, the drivers did not receive any requirements or tasks, allowing them to drive in their own driving style. The driver did not wear any device during the test to assure the authenticity of data acquisition. 
Table 1 Data acquisition sensor

\begin{tabular}{lll}
\hline Instrument name & Function & Technical index \\
\hline Millimeter-wave radar (ESR) & $\begin{array}{l}\text { Relative distance from the car } \\
\text { ahead, relative angle, relative speed } \\
\text { Video monitoring system }\end{array}$ & $\begin{array}{l}\text { Action monitoring of driver's foot, } \\
\text { operation behavior, and forward } \\
\text { and backward scenes }\end{array}$ \\
$\begin{array}{l}\text { Acquisition of the concrete location } \\
\text { of the test car and real-time speed } \\
\text { of the car }\end{array}$ & $\begin{array}{l}\text { Frequency is } 25 \mathrm{~Hz} \text { with synchronous } \\
\text { storage }\end{array}$ \\
Acquisition of the CAN data of the car & $\begin{array}{l}\text { Positioning precision is } 0.5 \mathrm{~m} \\
\text { CAN acquisition card }\end{array}$ & $\begin{array}{l}\text { Car speed is } 0.01 \mathrm{~km} / \mathrm{h} \text {; steering wheel } \\
\text { angle is } 1.0^{\circ} \text {, and the opening degree of } \\
\text { accelerator pedal is } 0.1 \% \text {. Braking signal } \\
\text { and turn lamp signal are acquired. }\end{array}$ \\
\hline
\end{tabular}

\subsection{SVM model}

SVM is a popular classification technology proposed by the AT\&T Bell Laboratory Research Group led by Vapnik in 1995. SVM is a supervised learning model based on learning algorithms, which is similar to neural network, but unlike neural networks, SVM uses mathematical methods and optimization techniques. It can be used in many fields, such as data processing, pattern recognition, and the analysis of classification and regression. Given a set of training samples and marking them into different categories, an SVM training model will assign new instances to one class or another, making it a non-probabilistic binary linear classification.

Several parameters would influence the driver's operation process when the brake lamps of the car ahead are on. A significant difference was observed in the mean value of some parameters in the states of slowing and not slowing down, but the difference of the mean value cannot be directly applied to the classification of the operation modes of the river. This finding is attributable to the fact that none of the parameters can directly decide whether the driver slowed down or not. However, a correlation exists between certain parameters, such as TTC, car-following time distance, and car-following distance, and whether the driver slowed down or not.

Analyzing the information perception process of the driver when the brake lamps of the car ahead are on shows that the driver should acquire the information of the moving state of his or her car and the car ahead. The driver should also obtain data from the surrounding traffic environment in a very short time and decide whether or

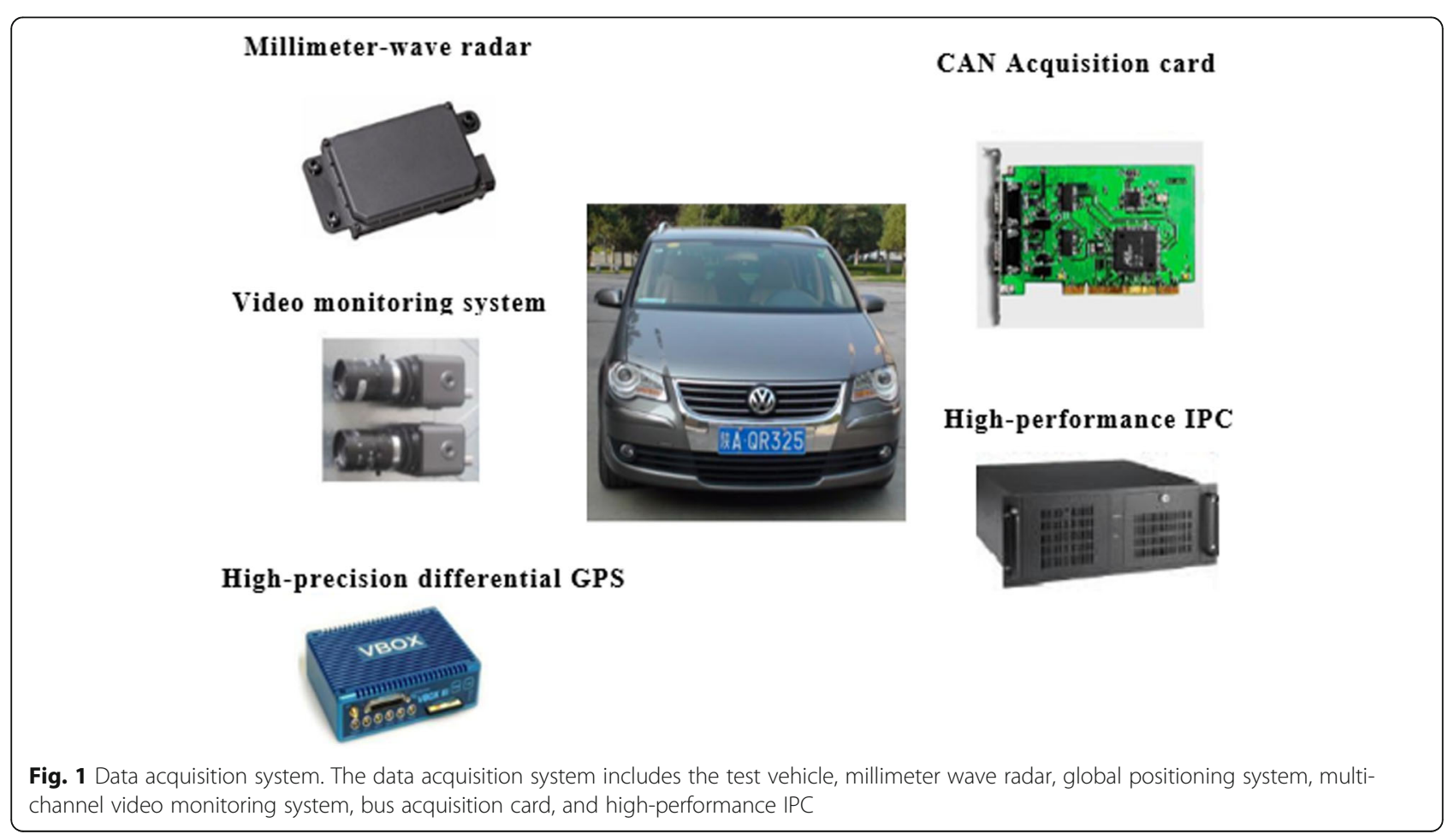


not to carry out the action. Simple mathematical equations cannot easily describe this process. A series of mode-recognition theories can be applied to solve these problems, such as neural network, fuzzy reasoning, convolutional neural network [42], and SVM. Considering the diversity of the parameter acquisition and the size of the sample, this study adopts the SVM theory to build the prediction model of the driver's operation. The best advantage of SVM is to introduce a multidimensional input parameter to a higher dimensional space and seek hyperplane to segment data input. The better the effect of data segmentation is, the more precise model recognition becomes.

The parameters that influence the operation model of the driver were used as input for the model. The operation behavior types of the driver were used as output. Tables 2 and 3 show the input and output, respectively.

The five kinds of input parameters are car-following time distance, car-following distance, speed of the car driven, relative speed, and TTC. State of output can be classified into four categories: keeping the accelerator pedal opening degree unchanged, loosening the accelerator pedal without braking, loosening the accelerator pedal and braking, and other special situations, which are represented as $0,1,2$, and, 3, respectively. The actual data collected by the millimeter-wave radar was frequently mixed with random noise because of the random disturbances that occurred during the measurement of the motion parameters of the leading vehicles. Therefore, the required data must be isolated from the original data. In this study, a discrete Kalman filter was used to filter the discrete data collected by the sensors.

The SVM tool cabinet was used in the MATLAB environment to train the data acquired in this study. About $60 \%$ of the data were randomly chosen from the 845 sets of data. The skilled samples were chosen as inputs in the tool cabinet for model training. The remaining $40 \%$ of the data were taken as the test samples to verify the effectiveness of the model.

\subsection{Data acquisition process}

The state of car following when the brake lamps of the car ahead are on can be acquired after the test by replaying the video monitoring system. Data at $10 \mathrm{~s}$ before and after the moment can be acquired, including the relative

Table 2 Model input

\begin{tabular}{ll}
\hline Input parameter & Unit \\
\hline Car-following time distance & $\mathrm{s}$ \\
Car-following distance & $\mathrm{m}$ \\
Speed of the car behind & $\mathrm{m} / \mathrm{s}$ \\
Relative speed & $\mathrm{m} / \mathrm{s}$ \\
TC & $\mathrm{s}$ \\
\hline
\end{tabular}

Table 3 Model output

\begin{tabular}{ll}
\hline Output state & Representation type \\
\hline $\begin{array}{l}\text { Keeping the accelerator pedal } \\
\text { opening degree unchanged }\end{array}$ & 0 \\
$\begin{array}{l}\text { Loosening the accelerator } \\
\text { pedal but without braking }\end{array}$ & 1 \\
Loosening the accelerator & 2 \\
pedal and braking & \\
Other special situations & 3
\end{tabular}

distance between the car driven and the car ahead, relative angle and speed, acquired car speed of CAN bus, opening degree of acceleration pedal, angle value of steering wheel, braking signal, and turn signal. Figure 2 shows a typical scene of brake lamps switched on.

The test processes of 20 participants were screened at 845 times of car following when the brake lamps were on. Data was correspondingly acquired. The operation behavior of the driver when the brake lamps of the car ahead were on was divided into four types. Table 4 shows the corresponding statistic frequencies.

Table 4 shows four kinds of situation when the brake lamps of the car ahead were on. The situation wherein the degree of opening of accelerator pedal remained unchanged was $53.4 \%$, maintained the highest proportion. An analysis of the corresponding state data of the car ahead indicated that the driver did not realize the need to reduce the speed when the car ahead brake slightly when the brake lamps of the car ahead were on. Thus, the driver maintained the opening degree of accelerator pedal and adopted further operation according to the risk degree of car following. Driving experience showed that the driver did not need to reduce the speed when the car ahead was braked slightly when the distance was relatively safe. This finding indicates that the data

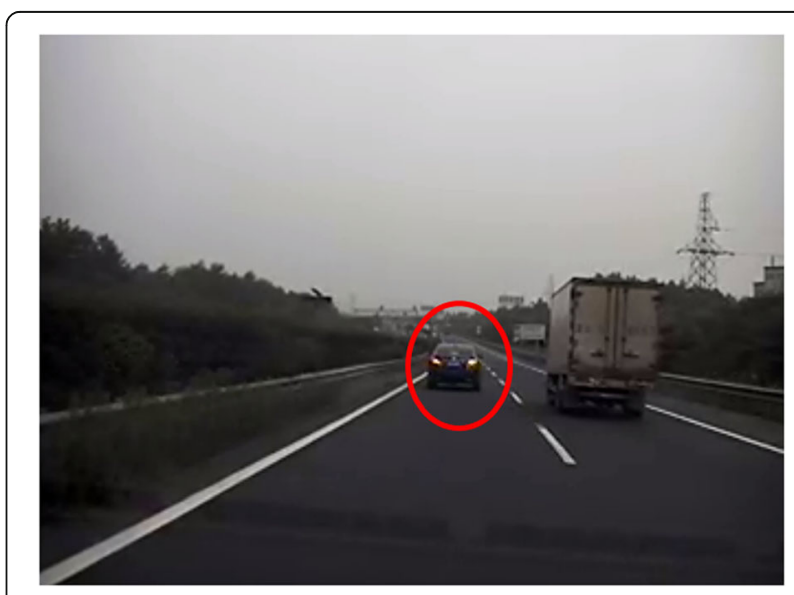

Fig. 2 Still image of the test car. A typical scene of brake lamps switched on of the preceding vehicle 
Table 4 Types of operation behavior of driver

\begin{tabular}{lll}
\hline State types & Operation of driver & Proportion (\%) \\
\hline State 0 & $\begin{array}{l}\text { Maintains the unchanged degree } \\
\text { of opening of accelerator pedal }\end{array}$ & 53.4 \\
State 1 & $\begin{array}{l}\text { Loosens the accelerator pedal } \\
\text { without braking }\end{array}$ & 26.8 \\
State 2 & $\begin{array}{l}\text { Loosens the accelerator pedal } \\
\text { and braking }\end{array}$ & 17.0 \\
State 3 & Other special situations & 2.8
\end{tabular}

acquired in this study are reasonable in terms of common sense.

When the risk degree of car following continues to increase, the driver would choose to loosen the accelerator pedal without braking. This state accounts for $26.8 \%$ of the total state types. After loosening the accelerator pedal, the car would slow down and the driver would seriously consider the risk degree of car following. If the risk degree continued to increase, the driver would choose to brake; otherwise, he or she could choose to loosen the accelerator pedal to ensure safety. When the risk degree of car following increased, the driver would directly choose to brake to avoid rear-end accident, accounting for $17.0 \%$. The proportion of other special situations is $2.8 \%$, which mainly includes the state of the driver's foot on the braking pedal (Fig. 3).

\section{Results analysis}

To investigate the driving behavior characteristics in car following when the brake lamps of the car ahead were on, this study used TTC, car-following time distance, speed of the car, and relative speed to analyze the operation of the driver when the brake lamps of the car ahead were on. This study examined the correlation between these parameters and the operation of the driver to support follow-up analysis and modeling. Finally, the

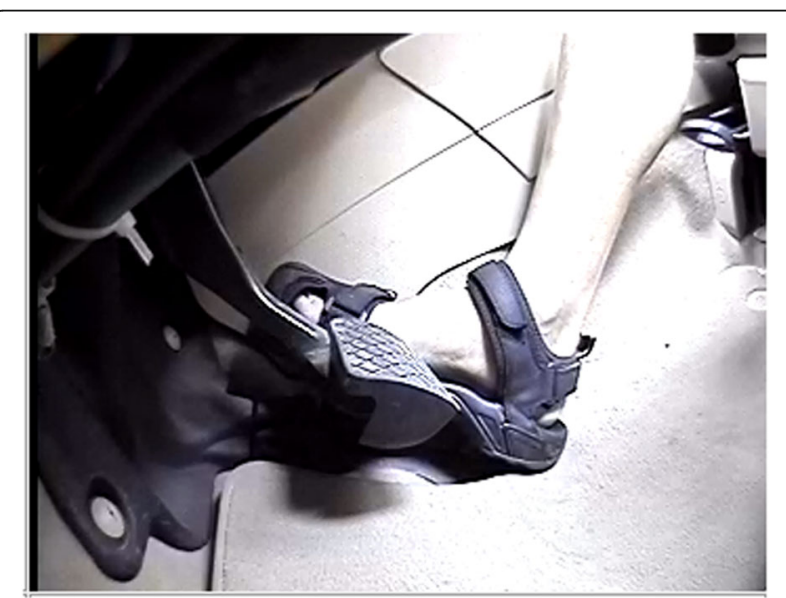

Fig. 3 Still image of the driver's foot. Driver's foot operation synchronized with typical scenes prediction accuracy of the behavior prediction model based on SVM was verified.

\subsection{TTC}

A TTC value at an instant $t$ is defined as the time that keeps until a collision occurs between two vehicles if the collision course and speed difference are maintained. The larger the TTC value is, the longer it is from the time of collision and the safer the driving is.

TTC value is the ratio of the distance between two cars and relative speed. Based on a laboratory test, Hirst and Graham found that when the TTC is set to $3 \mathrm{~s}$, the false alarm rate of the early warning system is low, and the driving safety can be effectively ensured [41]. When TTC $<0 \mathrm{~s}$, the speed of the car ahead is larger than the car behind it. Thus, collision will not occur, signifying that driving is safe. Related studies show that when $0 \mathrm{~s}<$ TTC $<3 \mathrm{~s}$, the distance between the two cars is too close and the car driving behind is faster than the car ahead. Collisions are likely to happen, which is a dangerous state. TTC $>3 \mathrm{~s}$ is a safe state. In this situation, the larger the value is, the higher the level of safety.

The TTC can be calculated with:

$$
\mathrm{TTC}=\frac{D}{V_{\text {host }}-V_{\text {ahead }}}
$$

where $V_{\text {host }}$ denotes the speed of the host vehicle, $V_{\text {ahead }}$ represents the speed of the vehicle ahead, and $D$ is the distance between the host vehicle and vehicle ahead.

In this paper, we recorded the TTC at the moment the brake lamps of vehicle ahead were on. The operation behaviors of the driver were divided into two types, namely, slowing down (including loosening of accelerator pedal and braking) and without slowing down. This study analyzed the relationship between the TTC parameter and whether the driver slowed down or not. After filtering data below 0,845 sets of data without those in special situations were classified for the TTC mean value and assessed whether or not they were in accelerating state. Figure 4 displays the results.

Figure 4 shows the large difference in TTC mean value between that in the accelerating state and nonaccelerating state. The TTC mean value of adopting braking is $3.3 \mathrm{~s}$, whereas that without slowing down is $20.3 \mathrm{~s}$. This difference is easily understood in terms of driving experience. However, an analysis of the TTC data shows that although both mean values differ, a large overlapping section continues to exist in the distribution range. For example, given that the TTC is $4.0 \mathrm{~s}$, the corresponding result of the data slows down, even though the results of other data do not. This result shows that the TTC parameter is unable to fully reflect whether the 


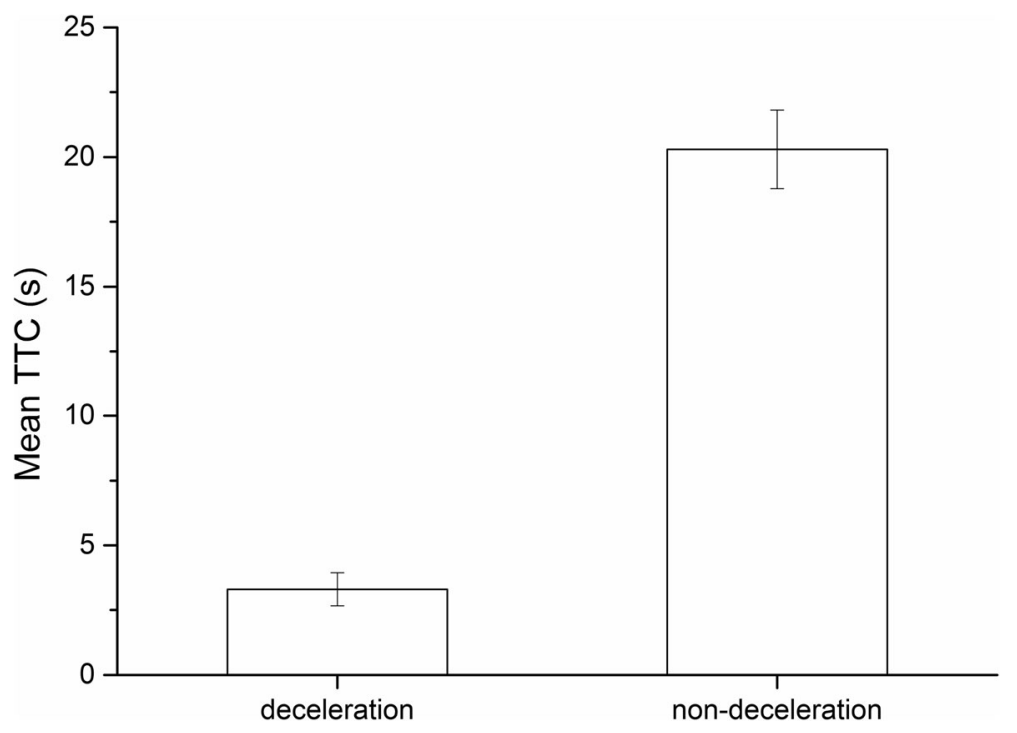

Fig. 4 Mean TTC in accelerating or non-accelerating state. The relationship between the TTC parameter and whether the driver slowed down or not was analyzed, and the TTC averages in different states are depicted. The TTC mean value of adopting braking is $3.3 \mathrm{~s}$, whereas that without slowing down is $20.3 \mathrm{~s}$

driver slows the car down or not. The precise threshold value cannot be acquired to distinguish the behavior of the driver. In terms of the actual perception of the driver, the driver besides TTC acquires other parameters and decides whether or not to slow down the car.

\subsection{Time distance of car following}

Car-following time distance refers to the division of distance between two cars by the speed of the car driven. The larger the car-following time distance is, the smaller the risk becomes for rear-end accidents. The mean value of time distance of car following in the slowing and nonslowing down states can be similarly acquired from the TTC parameter analysis method. Figure 5 displays the results.

In Fig. 5, a significant difference is found between carfollowing time distance in the two states. The mean value in the state of slowing down is $2.7 \mathrm{~s}$, whereas that in the state of non-slowing down is $9.2 \mathrm{~s}$. Similar to TTC distribution characteristics, a significant difference exists between the mean values of the parameter in the two states, but a certain threshold value cannot be clearly decided to distinguish the two kinds of data. Thus, an overlapping section exists between the two.

\subsection{Car-following distance}

Car-following distance directly influences driving safety. According to the regulations of "Road Traffic Safety Law," when the speed of a motor vehicle is higher than $100 \mathrm{~km} / \mathrm{h}$, the driver should keep a distance of above $100 \mathrm{~m}$ from the motor vehicle ahead. When the car speed is below $100 \mathrm{~km} / \mathrm{h}$, the distance from the car ahead in the same lane can be appropriately shortened with the minimum distance of no less than $50 \mathrm{~m}$. The theory of car following suggests a quantitative relationship when the distance between two cars is less than $125 \mathrm{~m}$ [20]. The greater the car-following distance is, the higher the level of driving safety becomes. By contrast, the smaller the car-following distance is, the higher the likelihood of a rear-end accident occurring.

Data for car-following distance were acquired when the brake lamps of the car ahead were on. The mean values of car-following distance in slowing and non-slowing down states were calculated. Figure 6 displays the results.

Figure 6 shows that the mean value of car-following distance in the slowing down state is $32.9 \mathrm{~m}$, whereas that in the state of non-slowing down is $51.3 \mathrm{~m}$. Compared with TTC and car-following distance, the distribution difference of car-following distance is smaller because car speed is not considered, proving to be an important factor influencing the car-following operation of the driver. Many overlapping data in the data distribution section exists in the two states. A certain threshold value of car-following distance cannot be established to distinguish whether the driver slows down or not.

\subsection{Verification of the prediction model}

The aim of training the SVM model is to acquire optimal parameters $c$ and $g$ of the kernel function, which directly decide on the classification of the input data by the kernel function. Although these two parameters are very important, there is not much guidance on how to determine them. The two parameters were constantly decided by the SVM in higher dimensional space through exploration until the 


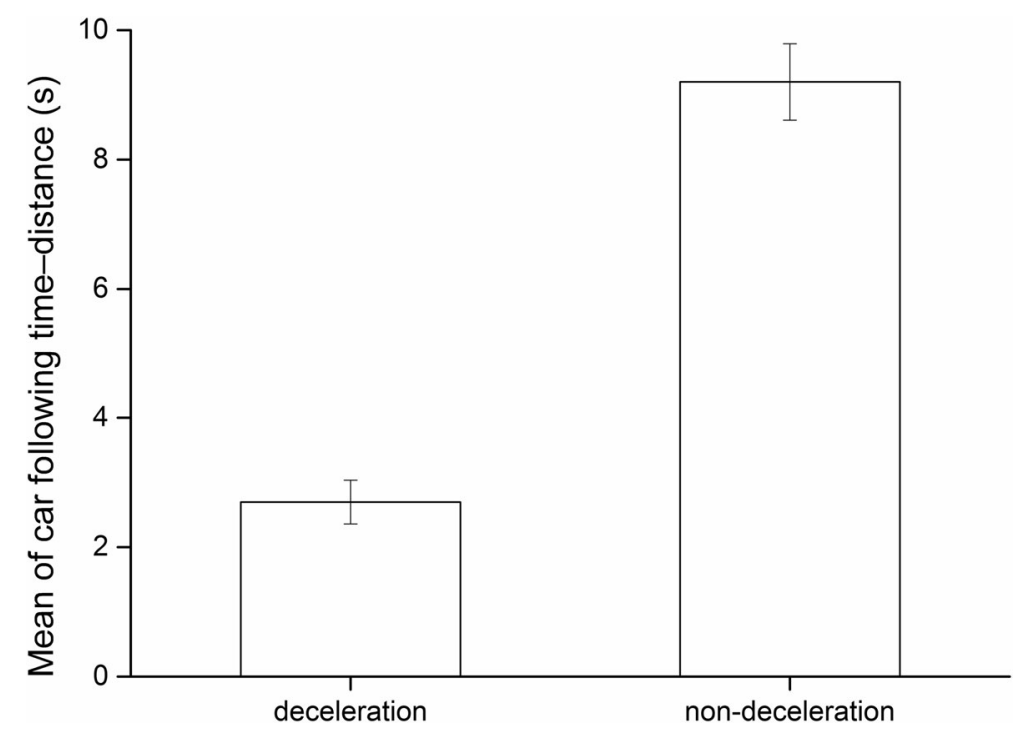

Fig. 5 Mean of car-following time distance in slowing or non-slowing down state. The mean value of car-following time distance in the state of slowing down is $2.7 \mathrm{~s}$, whereas that in the state of non-slowing down is $9.2 \mathrm{~s}$

input parameters attained optimal classification effect. After acquiring the optimal value of the two parameters, $40 \%$ of the remaining data were imported into the model to verify the precision of the operation mode prediction of the driver by the SVM model.

In Fig. 7, the line resembling a contour line is the high-dimensional segmentation curve of data acquired by the SVM model, which can divide the input data into different spaces. This plane figure can be converted into an output state data classification sketch map, which is easier to understand.
The overall prediction of precision values for the operation of the driver is distributed at approximately $80 \%$, with lamp difference in the prediction precision of different operation states. Some of the prediction results were converted into a $2 \mathrm{D}$ plane sketch map.

In Fig. 9, the desired output is the corresponding operation state of the driver within the acquired samples, whereas the predicted output is the actual output result of the SVM model. The vertical axis represents the four different types of driving behaviors when confronted by the braking behavior of the preceding vehicle. By

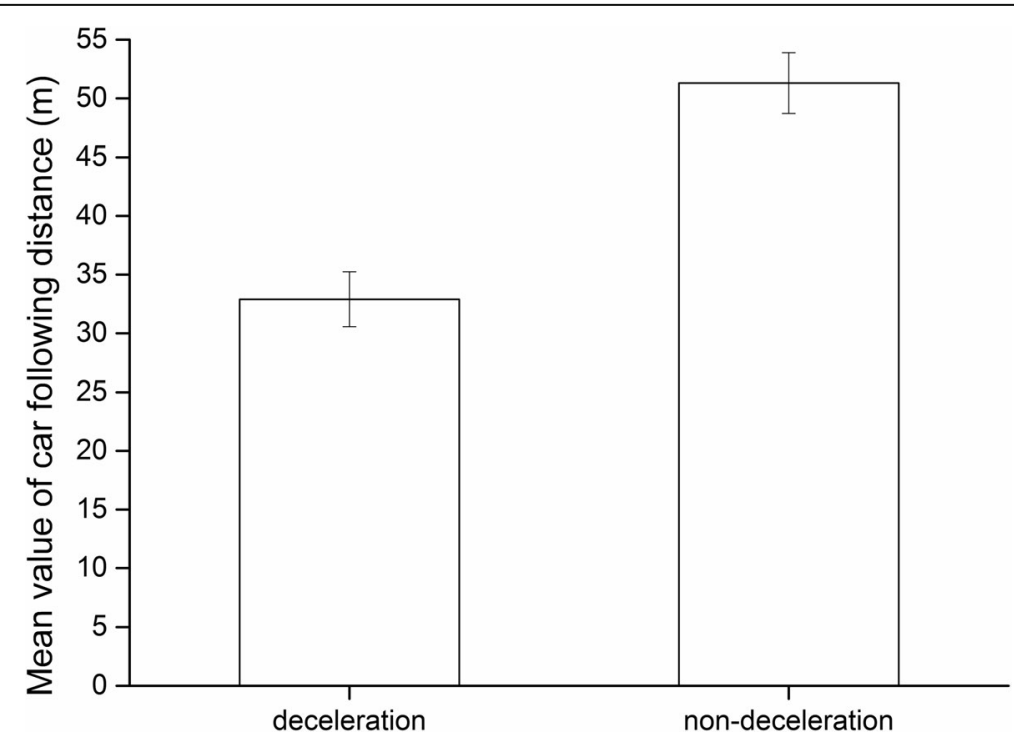

Fig. 6 Mean of car-following distance in slowing down state or not. The mean value of car-following distance in the slowing down state is 32.9 $\mathrm{m}$, whereas that in the state of non-slowing down is $51.3 \mathrm{~m}$ 


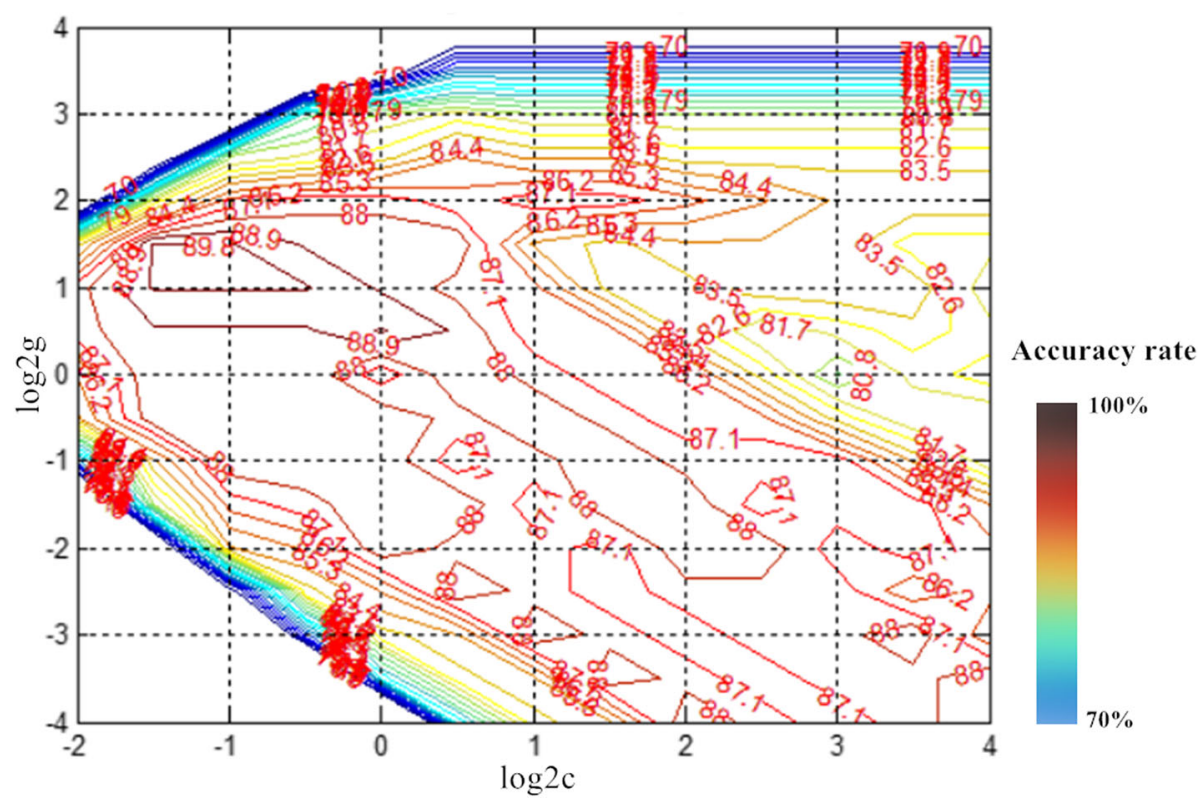

Fig. 7 Classification result of the SVM model. The line resembling a contour line is the high-dimensional segmentation curve of data acquired by the SVM model, which can divide the input data into different spaces

combining Figs. 8 and 9, the SVM model precisely classified most of the data, but differences still exist in the predicted and actual result for some of the data. The ROC curve was used to analyze the overall prediction accuracy of the SVM model and evaluate the prediction efficiency of the SVM model [43].
Figure 10 shows the prediction result of the driver's operation in the ROC curve under different falsepositive rates. Great changes are observed in the prediction accuracy of the model. By taking the false-positive rate of $5 \%$ as reference, the corresponding prediction accuracy of the model is determined at $80.8 \%$.

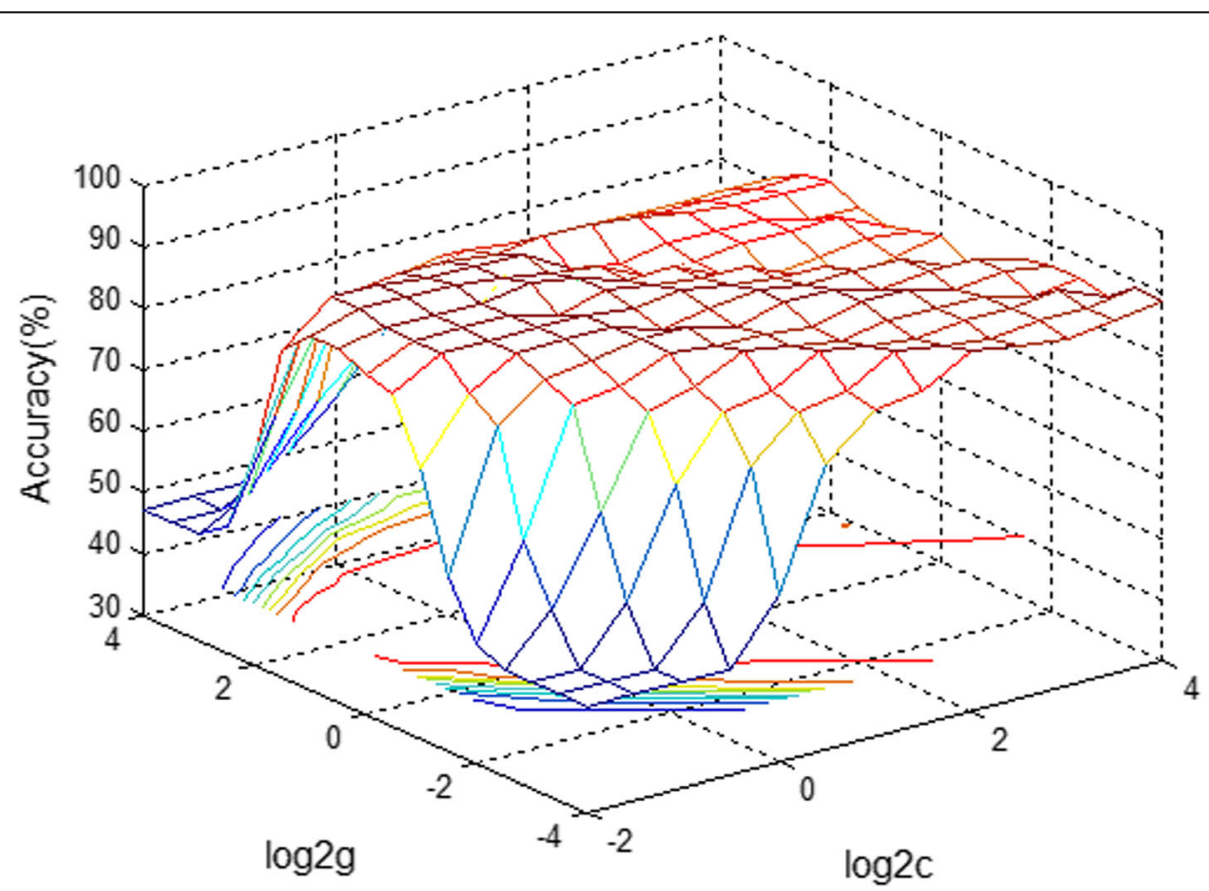

Fig. 8 Prediction result of the driver's operation state. The overall prediction accuracy values for the operation of the driver under different parameters 


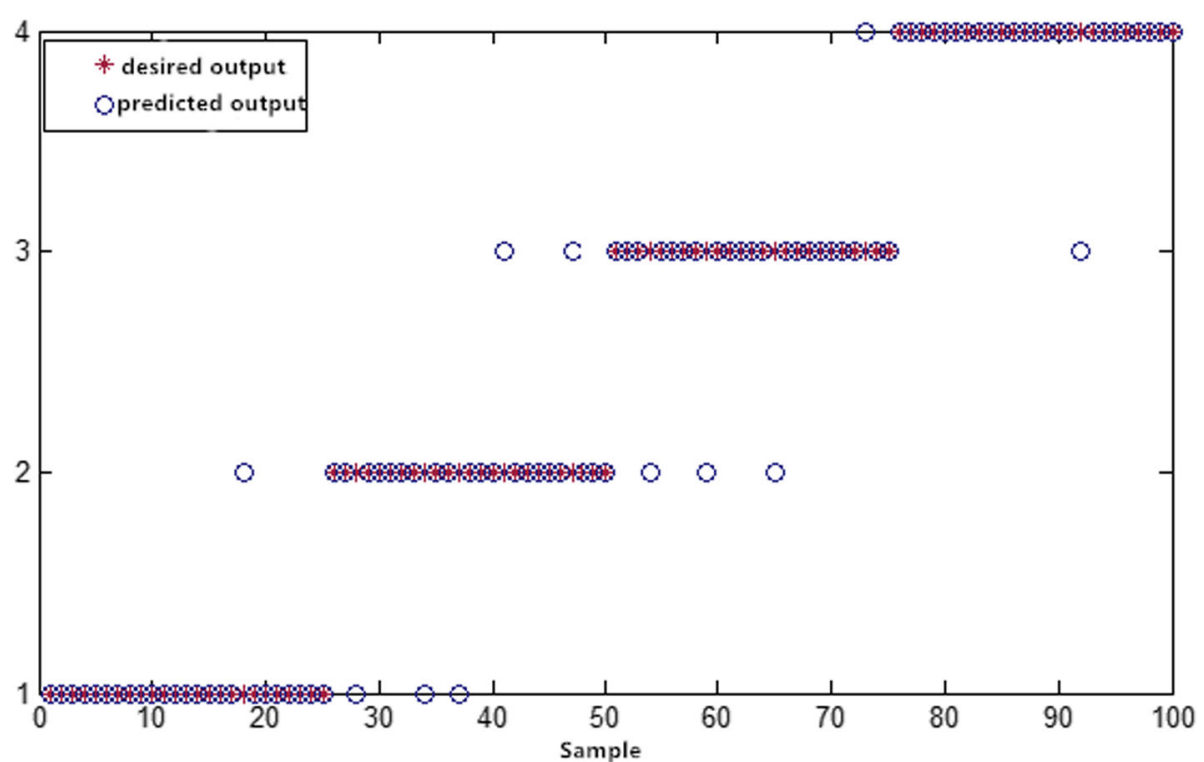

Fig. 9 Prediction result of the driver's operation state. The desired output is the corresponding operation state of the driver within the acquired samples, whereas the predicted output is the actual output result of the SVM model

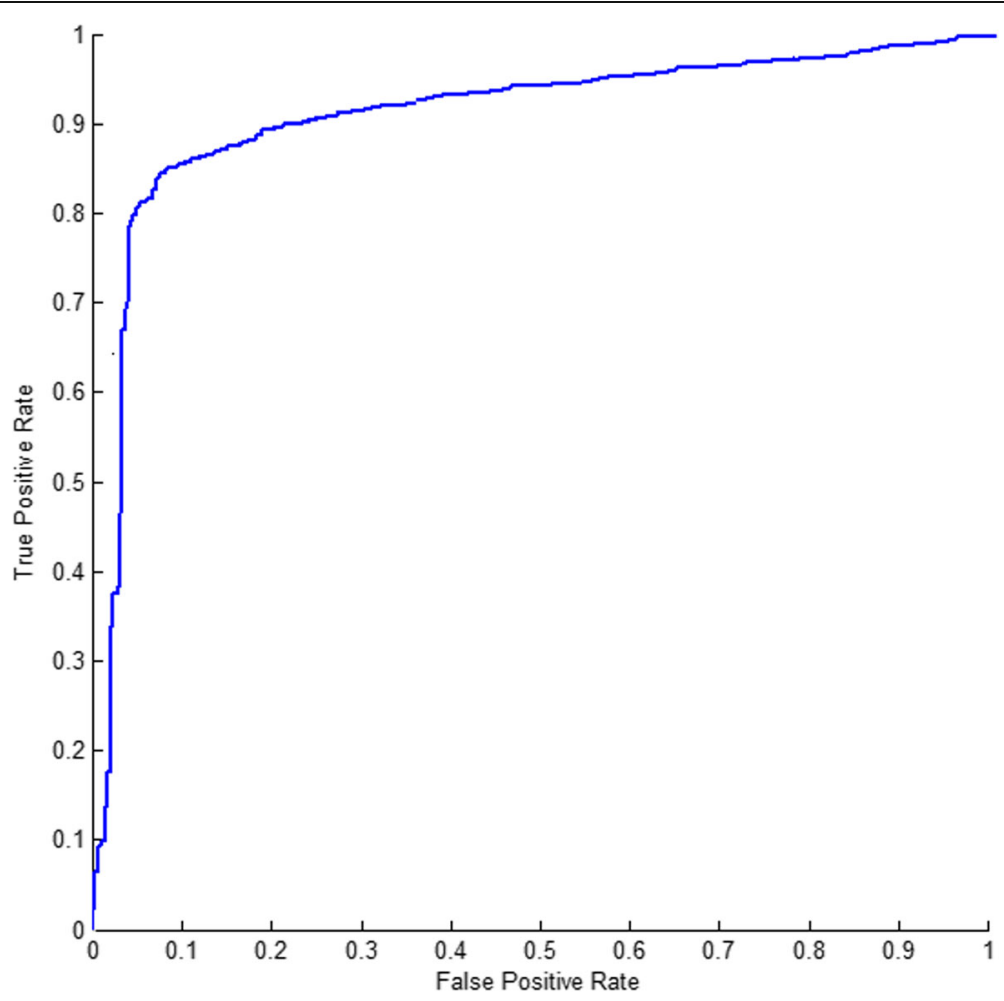

Fig. 10 ROC curve for evaluating the prediction model accuracy. The ROC curve was used to analyze the overall prediction accuracy of the SVM model and evaluate the prediction efficiency of the SVM model. By taking the false-positive rate of $5 \%$ as reference, the corresponding prediction accuracy of the model is determined at $80.8 \%$ 


\section{Discussion}

Past studies on car-following model used different methods to model, analyze, and predict the operation behavior of the driver during car following [10, 11, 36]. These studies figured out the relationship of movement states between host car and car ahead; however, the impact of psychological characteristics was underestimated. Due to sensation seeking [44], some drivers prefer a risky follow-up. In this case, it is a dangerous follow-up based on TTC and THW method. However, the driver usually thinks the preceding vehicle will not brake urgently and considers their driving state is safe. Once the brake lamps of car ahead turn on, driver's alertness will rise significantly, which is significantly different from the traditional car model. When the brake lamps turn on, the driver will make a different response based on the relationship of movement state between the host car and the car ahead. This process is different from the traditional follow-up method. Therefore, the model in this paper focuses on the decision-making process of driving behavior when the front brake lamps are turned on. There is an example of a typical application scenario: The driver ignores the light lamps of car ahead turned on while distracted driving, advanced driver assistance systems (ADAS) detect the information and predict the driver should brake, but the driver does not take any operation. In this case, ADAS can judge the driving state is dangerous and directly control the host car. This will reduce false alarms while ensuring safety.

The chosen parameters included the moving state of the car driven, the car ahead, and the surrounding traffic environment. For instance, the distance between cars, speed of the car driven, relative car speed, TTC, and THW were used as input variables to predict the safety of the car-following process with a prediction accuracy of $90.2 \%$ [10]. Compared with the results of other similar methods, $90.2 \%$ is not a very high value. However, the driver's behavior in car following is a perception decision-making process with quick and multi-source information input, which is random for drivers and driver groups. This phenomenon is especially true after the study period is shortened.

Jiang et al. divided the driver behavior into two types, whereas the present study divides the results into four types [10]. The recognition accuracy of the behavior prediction model with two kinds of classification is better than that of prediction model with four kinds of classification. However, the present study mainly analyzes the actual operation behavior when the brake lamps of the car ahead were on, such as when the driver loosens the accelerator pedal without braking. In this case, the driver adopts the slowing down method but with poor effect. In terms of the psychological perception process and safety control of the driver, the psychological safety of the driver quickly increases after loosening the accelerator pedal. Although the speed of the vehicle slowly decreases, braking is needed to enable the driver to apply methods immediately and ensure safe car following. Therefore, though this study has divided the operation modes into four types with an overall recognition rate of only $80.8 \%$, the explaining effect of the model to the behavior of the driver resembles actual situations. By considering the rear-end collision alarm system, operation mode types should be reduced into two to increase the recognition accuracy rate of the model.

\section{Conclusion}

An actual road test was conducted to acquire the moving state data of a car driven when the brake lamps of the car ahead were on during car following using moving the state data of the car ahead, traffic environment data, and the operation data of the driver. The distribution characteristics of car-following time distance, carfollowing distance, and TTC parameter were analyzed based on whether or not the driver slowed down. Significant differences were observed in the three parameters of slowing down and non-slowing down modes. However, high overlap ratio exists in the distribution range, resulting in the difficulty of distinguishing and predicting the operation modes of the driver.

According to the car-following time distance, carfollowing distance, speed of the car ahead, relative speed, and TTC, a driving behavior prediction model was provided with the SVM. When the brake lamps of the car ahead were switched on, the reaction behavior of the driver was divided into four types: keeping the accelerator pedal opening degree unchanged, loosening the accelerator pedal but without braking, braking, and other special situations. The SVM method was implemented to build a prediction model of the operation behavior of the driver. The input variables of car-following time distance, car-following distance, speed of the driver's car, relative speed, and TTC were utilized. Although the ultimate prediction accuracy rate is only $80.8 \%$, this model resembles real-life situations and reflects the decisionmaking process of the driver.

This model can help the rear-end collision warning system to predict the behavior of the driver, select the proper warning time according to different behaviors of the driver, and improve the effectiveness of the early warning system while reducing the false alarm rate.

\section{Authors' contributions \\ HW and CW conceptualized the idea and designed the experiments. MIG and SbW participated in the data processing. HW and MIG contributed in model training. HW contributed in writing and draft preparation, and CW supervised the research. All authors read and approved the final manuscript.}

\section{Funding}

The study was supported by National Natural Science Foundation of China (51908054). 


\section{Availability of data and materials}

Not applicable.

\section{Competing interest}

The authors declare that they have no competing interests.

\section{Received: 4 October 2019 Accepted: 31 December 2019}

Published online: 08 January 2020

\section{References}

1. Z. Zheng, M. Sarvi, Modeling, calibrating, and validating car following and lane changing behavior. Transportation Research Part C: Emerging Technologies 71, 182-183 (2016)

2. R. Hoogendoorn et al., Mental workload, longitudinal driving behavior, and adequacy of car-following models for incidents in other driving lane. Transportation Research Record 2188(1), 64-73 (2010) https:/doi.org/10.3141/2188-08

3. A. Tang, A. Yip, Collision avoidance timing analysis of DSRC-based vehicles. Accident Analysis \& Prevention 42(1), 182-195 (2010) https://doi.org/10. 1016/j.aap.2009.07.019

4. Kometani, E. "Dynamic behavior of traffic with a nonlinear spacing-speed relationship." Theory of Traffic Flow (Proc. of Sym. on TTF (GM)) (1959): 105-119.

5. R.E. Chandler, R. Herman, E.W. Montroll, Traffic dynamics: studies in car following Operations research 6(2), 165-184 (1958) https://doi.org/10.1287/opre.6.2.165

6. J. Treiterer, J. Myers, The hysteresis phenomenon in traffic flow. Transportation and traffic theory 6, 13-38 (1974)

7. Aron, Maurice. "Car following in an urban network: simulation and experiment. " Proceedings of Seminar D, 16^<th> PTRC Meeting, 1988. 1988.

8. W. Helly, Simulation of bottlenecks in single-lane traffic flow (1959)

9. Hidas, Peter. "A car-following model for urban traffic simulation." Traffic engineering \& control 39.5 (1998).

10. R. Jiang, M.B. Hu, H.M. Zhang, et al., On some experimental features of carfollowing behavior and how to model them. Transportation Research Part B: Methodological 80, 338-354 (2015) https://doi.org/10.1016/j.trb.2015.08.003

11. Tang T Q, Zhang J, Chen L. "Analysis of vehicle's safety envelope under carfollowing model." Physica A: Statistical Mechanics and its Applications, 2017,474, 127-133. https://doi.org/10.1016/j.physa.2017.01.076

12. P.G. Gipps, A behavioural car-following model for computer simulation. Transportation Research Part B: Methodological 15(2), 105-111 (1981) https://doi.org/10.1016/0191-2615(81)90037-0

13. Zhou T, Sun D, Kang Y, et al. "A new car-following model with consideration of the prevision driving behavior." Communications in Nonlinear Science and Numerical Simulation ,2014,19(10): 3820-3826. https://doi.org/10.1016/j.cnsns.2014.03.012

14. Y. Yang, K. Wada, T. Oguchi, et al., Variability of observed drivers' carfollowing behavior on expressway basic segment. Transportation Research Procedia 25, 1503-1532 (2017) https://doi.org/10.1016/j.trpro.2017.05.179

15. A. Tordeux, S. Lassarre, M. Roussignol, An adaptive time gap car-following model. Transportation Research Part B Methodological 44(8-9), 1115-1131 (2010) https://doi.org/10.1016/j.trb.2009.12.018

16. T.Q. Tang, W.F. Shi, H.Y. Shang, An extended car-following model with consideration of the reliability of inter-vehicle communication. Measurement 58(11), 286-293 (2014) https://doi.org/10.1016/j.measurement.2014.08.051

17. B. Simons-Morton, N. Lerner, J. Singer, The observed effects of teenage passengers on the risky driving behavior of teenage drivers. Accident Analysis \& Prevention 37(6), 973-982 (2005) https://doi.org/10.1016/j.aap. 2005.04.014

18. Trnros, J., et al. "Effects of ACC on driver behaviour, workload and acceptance in relation to minimum time headway." 9th World Congress on Intelligent Transport SystemsITS America, ITS Japan, ERTICO (Intelligent Transport Systems and Services-Europe). 2002.

19. Varotto, Silvia F., et al. "Empirical longitudinal driving behavior in authority transitions between adaptive cruise control and manual driving." Transportation Research Record: Journal of the Transportation Research Board 2489 (2015): 105-114. https://doi.org/10.3141/2489-12

20. DeLucia P R, Mather R D. "Motion extrapolation of car-following scenes in younger and older drivers." Human factors ,2008,48(4): 666-674. https://doi. org/10.1518/001872006779166352

21. Egelund N. "Spectral analysis of heart rate variability as an indicator of driver fatigue." Ergonomics,1982, 25.7: 663-672.

22. Lamble D, Kauranen T, Laakso M, et al. "Cognitive load and detection thresholds in car following situations: safety implications for using mobile (cellular) telephones while driving." Accident Analysis \& Prevention,1999, 31(6): 617-623. https://doi.org/10.1016/S0001-4575(99)00018-4

23. D. Stavrinos, J.L. Jones, A.A. Garner, et al., Impact of distracted driving on safety and traffic flow. Accident Analysis \& Prevention 61, 63-70 (2013) https://doi.org/10.1016/j.aap.2013.02.003

24. Z. Gao, D.Y. Wang, S.H. Wan, et al., Cognitive-inspired class-statistic matching with triple-constrain for camera free 3D object retrieval. Future Generation Computer Systems 94, 641-653 (2019) https://doi.org/10.1016/jfuture.2018.12.039

25. Strayer D L, Drew F A. "Profiles in driver distraction: effects of cell phone conversations on younger and older drivers." Human factors, 2004,46(4): 640-649. https://doi.org/10.1518/hfes.46.4.640.56806

26. J.B. Sheu, H.J. Wu, Driver perception uncertainty in perceived relative speed and reaction time in car following-a quantum optical flow perspective. Transportation Research Part B: Methodological 80, 257-274 (2015) https:// doi.org/10.1016/j.trb.2015.07.017

27. Li S, Yang L, Gao Z,et al. "Stabilization strategies of a general nonlinear carfollowing model with varying reaction-time delay of the drivers." ISA transactions, 2014, 53(6), 1739-1745. https://doi.org/10.1016/j.isatra.2014.08.017

28. Gao Z, Xuan HZ, Zhang $\mathrm{H}$,et al. "Adaptive fusion and category-level dictionary learning model for multi-view human action recognition." IEEE Internet of Things Journal, 2019. https://doi.org/https://doi.org/10.1109/JOT. 2019.2911669

29. H. Alm, L. Nilsson, The effects of a mobile telephone task on driver behaviour in a car following situation. Accident Analysis \& Prevention 27(5), 707-715 (1995) https://doi.org/10.1016/0001-4575(95)00026-V

30. S. Wan, Y. Zhao, T. Wang, et al., Multi-dimensional data indexing and range query processing via Voronoi diagram for internet of things. Future Generation Computer Systems 91, 382-391 (2019) https://doi.org/10.1016/j.future.2018.08.007

31. T. Wakita et al., Driver identification using driving behavior signals. IEICE TRANSACTIONS on Information and Systems 89(3), 1188-1194 (2006)

32. Zhang $R$, Xie P, Wang C, et al. "Classifying transportation mode and speed from trajectory data via deep multi-scale learning." Computer Networks 162 (2019). https://doi.org/10.1016/j.comnet.2019.106861

33. P. Bian, W. Li, Y. Jin, et al., Ensemble feature learning for material recognition with convolutional neural networks. EURASIP Journal on Image and Video Processing 2018, 64 (2018)

34. N. Davoodi, A.R. Soheili, S.M. Hashemi, A macro-model for traffic flow with consideration of driver's reaction time and distance. Nonlinear Dynamics 83(3), 1621-1628 (2016)

35. S. Wan, Z. Gu, Q. Ni, Cognitive computing and wireless communications on the edge for healthcare service robots. Computer Communications (2019) https://doi.org/10.1016/j.comcom.2019.10.012

36. X. Xu, Y. Xue, L. Qi, et al., An edge computing-enabled computation offloading method with privacy preservation for internet of connected vehicles. Future Generation Computer Systems 96, 89-100 (2019)

37. H. Qian, Y. Ou, X. Wu, et al., Support vector machine for behavior-based driver identification system. Journal of Robotics 2010 (2010)

38. Y. Zhao, H. Li, S. Wan, et al., Knowledge-aided convolutional neural network for small organ segmentation. IEEE journal of biomedical and health informatics 23(4), 1363-1373 (2019) https://doi.org/10.1109/JBHI.2019.2891526

39. S. Ding, S. Qu, Y. Xi, et al., Stimulus-driven and concept-driven analysis for image caption generation. Neurocomputing (2019)

40. Zhang J, Suzuki K, Fujiita M. "Car-following behavior with instantaneous driver-vehicle reaction delay: a neural-network-based methodology." Transportation Research Part C Emerging Technologies, 2013, 36(11):339351. https://doi.org/10.1016/j.trc.2013.09.010

41. Hirst, Stephen, and Robert Graham. "The format and presentation of collision warnings. Ergonomics and safety of intelligent driver interfaces." (1997): 203-219.

42. S.H. Wan, S. Goudos, Faster R-CNN for multi-class fruit detection using a robotic vision system. Computer Networks 107036 (2019) https://doi.org/10. 1016/..comnet.2019.107036

43. D.J. Sebald, J.A. Bucklew, Support vector machine techniques for nonlinear equalization. IEEE Transactions on Signal Processing 48(11), 3217-3226 (2000) https://doi.org/10.1109/78.875477

44. $\mathrm{Xu} X, \mathrm{Li} Y$ and Jin $Y$. "Hierarchical discriminant feature learning for crossmodal face recognition." Multimedia Tools and Applications. https://doi.org/ 10.1007/s11042-019-7683-4

\section{Publisher's Note}

Springer Nature remains neutral with regard to jurisdictional claims in published maps and institutional affiliations. 